\title{
Influence of the pressure-dependent resonance frequency on the bifurcation structure and backscattered pressure of ultrasound contrast agents: a numerical investigation
}

\author{
Amin Jafari Sojahrood • Omar Falou • Robert Earl • \\ Raffi Karshafian - Michael C. Kolios
}

Received: 1 May 2014 / Accepted: 7 January 2015 / Published online: 31 January 2015

(C) Springer Science+Business Media Dordrecht 2015

\begin{abstract}
The bifurcation structure of the oscillations of ultrasound contrast agents (UCAs) was studied as a function of the driving pressure for excitation frequencies that were determined using the UCAs pressuredependent resonances $\left(f_{\mathrm{s}}\right)$. It was shown that when excited by the $\left(f_{\mathrm{s}}\right)$, the UCA can undergo a saddlenode bifurcation (SNB) to higher amplitude oscillations. The driving pressure at which the SNB occurs is controllable and depends on the $\left(f_{\mathrm{s}}\right)$ magnitude. Utilizing the appropriate $\left(f_{\mathrm{s}}\right)$, the scattering cross section of the UCAs can significantly be enhanced (e.g., $\sim$ ninefold) while at the same time avoiding potential UCA destruction (by limiting the radial expansion ratio $<2$ ). This offers significant advantages for optimizing UCA-mediated imaging and therapeutic ultrasound applications.
\end{abstract}

Keywords Ultrasound contrast agents .

Microbubbles · Ultrasound · Bifurcation structure · Pressure-dependent resonance $\cdot$ Nonlinear backscatter pressure $\cdot$ Nondestructive oscillations

A. J. Sojahrood $(\varangle) \cdot$ O. Falou · R. Earl · R. Karshafian · M. C. Kolios Department of Physics, Ryerson University, Toronto, ON, Canada

e-mail: amin.jafarisojahrood@ryerson.ca

\section{Introduction}

Bubbles excited by ultrasound waves are highly nonlinear oscillators [1]. It has been shown both numerically and experimentally that bubbles exhibit complex and chaotic dynamics [1-11]. Bubbles encapsulated by a shell are being used in diagnostic ultrasound as contrast agents (UCAs) [12]. The dynamics of the UCAs has also been associated with complex and chaotic oscillations $[8,11]$.

Despite the complex behavior, bubbles and UCAs have become instrumental in a wide variety of fields. UCAs are used in sonoluminscence [6], in sonochemistry to increase chemical yields [13], and in material science [14]. In the context of medical ultrasound, studies have shown the potential of the UCAs to target and enhance drug/gene delivery [15], reversibly open blood-brain barrier to deliver drugs to the brain [16] and in ultrasound diagnostic imaging to enhance the detection of cancer even at early stages and with molecular sensitivity [17].

Despite many applications of UCAs, the relationship between the ultrasound exposure parameters (e.g., frequency and pressure) and the UCA behavior is not well understood. The UCA response to ultrasound is nonlinear and complex; thus, the exposure conditions in some applications may not be optimized. A comprehensive understanding of the dynamics of the UCAs for the large range of relevant exposure parameters can be achieved using methods of nonlinear physics. 
Fundamental insights into the dynamical properties of the UCAs can lead to optimization of the exposure parameters for particular applications. However, many of the numerical and experimental studies on the UCA dynamics have been carried out for a limited range of ultrasound frequencies and pressures. Due to the complexity and sensitivity of the exposure and the UCA parameters on the UCA oscillation dynamics, the limited information acquired in these studies does not provide a comprehensive framework that can be used further for the unification and classification of the UCAs dynamics. Furthermore, many potential exposure parameter combinations may be excluded due to the discrete nature of the parameters that have been investigated.

In most UCA applications, resonant UCA oscillations are of fundamental importance as they result in the highest energy transfer from the ultrasonic field to the UCAs [18]. As an example, in ultrasonic imaging, when UCAs are driven with their resonance frequency, they generate a significant backscattered signal which will enhance the signal compared with the background signal [19]. Previous studies for free [20] and encapsulated [21-24] bubbles have shown a shift in the UCA resonance occurs for different driving ultrasound amplitudes. Higher driving pressures have shown to decrease the resonance frequency of the free and encapsulated bubbles [20-24]. Despite the wellknown fact of this shift in the resonant frequency for free bubbles [2] and the recent detailed observations of this shift for contrast agents [21-24], to our best knowledge, no study has been published that examines the consequences of this shift in the UCA resonant frequency on the dynamical behavior of the UCAs. The dynamics of the system over a wide continuous range of the pressure and frequency has not been studied. To address the effect of the excitation frequency and the applied acoustic pressure on the dynamics of the UCAs, these two parameters must be investigated together, taking into account the nonlinear relation between the acoustic pressure and the UCA resonance frequency.

The focus of this study is to investigate the dynamics of the resonant UCAs (excited by linear and pressuredependent resonance frequencies). The aim of this work is to build a fundamental understanding of the dynamics of the ultrasound contrast agents for the identification and classification of the nonlinear signatures of the resonant UCAs. This is studied firstly by calculat- ing the UCA resonance curves at different ultrasounddriving pressures. In the second step, these resonance frequencies are used to drive the UCA excitation and the dynamical behavior of the UCAs are visualized by the aid of the bifurcation diagrams of the radial oscillations of the UCAs as a function of the driving acoustic pressures. In the third step, the maximum backscattered pressure is calculated with the aid of bifurcation diagrams to investigate the effect of the nonlinear resonance on the backscattered pressure in the regime of nondestructive UCA oscillations.

\section{Methods}

\subsection{The bubble model}

The radial oscillations of the UCAs were simulated by solving the Church-Hoff model [25] for UCAs through applying the fourth-order Runge-Kutta technique:

$$
\begin{aligned}
\rho\left(\ddot{R} R+\frac{3}{2}\right) \dot{R}^{2}= & P_{0}\left(\left(\frac{R_{0}}{R}\right)^{3 \Gamma}-1\right) \\
& -p_{\mathrm{A}}(t)-4 \mu_{\mathrm{L}} \frac{\dot{R}}{R}-12 \mu_{\mathrm{s}} \theta \frac{R_{0}^{2}}{R^{3}} \frac{\dot{R}}{R} \\
& -12 G_{\mathrm{s}} \theta \frac{R_{0}^{2}}{R^{3}}\left(1-\frac{R_{0}}{R}\right)
\end{aligned}
$$

In this equation, $R$ is the UCA radius at time $t, R_{0}$ is the initial radius of the unexcited UCA, $\dot{R}$ is the UCA wall velocity at time $t, \ddot{R}$ is the UCA wall acceleration at time $t, \rho$ is the density of liquid and is equal to $998 \mathrm{~kg} / \mathrm{m}^{3}$ and $\mu_{\mathrm{L}}$ is the density of the surrounding liquid which is equal to $0.001 \mathrm{~N} \mathrm{~s} / \mathrm{m}^{2}, P_{0}$ is the equilibrium gas pressure within the bubble which is $1.01 \times 10^{5} \mathrm{~Pa}, \Gamma$ is polytropic exponent which is equal to 1.095 for $\mathrm{SF}_{6}$. The symbol $\mu_{\mathrm{s}}$ stands for the shell viscosity, $\theta$ is the shell thickness, and $G_{\mathrm{s}}$ is the shear modulus of the shell. The values for these parameters are given in Sect. 2.2. The driving ultrasound pulse, $p_{\mathrm{A}}(t)$, is a sinusoidal signal consisting of 80 cycles at varying acoustic pressure amplitudes $(1 \mathrm{kPa}-1 \mathrm{MPa})$ and frequencies $(0.5-10 \mathrm{MHz})$ :

$p_{\mathrm{A}}(t)=P_{\mathrm{A}} \sin (2 \pi f t)$

In the above equation, $p_{\mathrm{A}}(t)$ is the driving sound field where $P_{\mathrm{A}}$ is the acoustic pressure amplitude, $f$ is the driving frequency and $t$ is time. In addition, the 
backscattered acoustic pressure $\left(P_{\mathrm{sc}}\right)$ at a distance $d$ from the center of the UCA, was calculated using [26]:

$P_{\mathrm{sc}}=\rho_{\mathrm{L}} \frac{R}{d}\left(2 \dot{R}^{2}+R \ddot{R}\right)$

In this paper, the backscatter pressure of different UCAs was calculated at a normalized distance $d=1$ as discussed in [27]. The maximum scattering cross section was calculated using [28]:

$\mathrm{SCS}_{\max }=\left(4 \pi R_{0}^{2}\right)\left|\frac{P_{\mathrm{sc}}}{P_{\mathrm{A}}}\right|_{\text {max }}^{2}$

where $\mathrm{SCS}_{\max }$ is the maximum scattering cross section.

\subsection{Simulation parameters}

Four UCAs with initial radii of $1,2,3$, and $4 \mu \mathrm{m}$ were considered. The sample shell parameters of $G_{\mathrm{s}}=$ $50 \mathrm{MPa}, \theta=4 \mathrm{~nm}$ and $\mu_{\mathrm{sh}}=\frac{1.49\left(R_{0}(\mu \mathrm{m})-0.86\right)}{\theta(\mathrm{nm})}$ were chosen. The shell viscosity was based on the values reported in [29] that take into account the increase in the shell viscosity for larger UCAs. These parameters were chosen based on published values for a generic UCA; this is done since the main purpose of the paper is to investigate a general characteristic of the UCA system excited by its pressure-dependent resonance frequency and not the behavior of one particular UCA. The results may therefore be generalized to all the UCAs whose dynamics are governed by equations like Eq. 1.

\section{Results}

\subsection{Pressure-dependent resonance}

First the resonance frequencies of the UCAs were calculated by solving Eq. 1. For each UCA, the primary linear resonance frequency was calculated by assuming a weak driving pressure amplitude of $P_{\mathrm{A}}=1 \mathrm{kPa}$. To calculate the maximum amplitude of the radial oscillations, the last 40 cycles of a 80 -cycle sonication were used. In practice attenuation measurements are used to determine the resonance frequency of the UCAs [23]. In these experiments, the ultrasound pulse hits the UCAs which are initially at rest. Thus, at each frequency step $(0.01 \mathrm{MHz})$, the Hoff model was solved with the initial conditions (ICs) of $R(t=0)=R_{0}$ and $\dot{R}(t=0)=0$ to emulate UCA experimental conditions. The dynamics of a highly nonlinear system like UCA is strongly dependent on the ICs [7,30]. The effect of the ICs on the resonance curves is studied in Sect. 4. After calculating the linear resonance frequency for $P_{\mathrm{A}}=1 \mathrm{kPa}$, for each UCA, the $P_{\mathrm{A}}$ was increased and the new resonance frequencies were calculated for five different $P_{\text {As }}$. Again at each frequency step, the ICs is set as $R(t=0)=R_{0}$ and $\dot{R}(t=0)=0$. The maximum pressure amplitude was set based on a maximum ratio of the radial oscillations of the UCA of $R_{n}=1.9\left(R_{n}=\frac{R_{\max }}{R_{0}}\right)$. This upper limit was used since the UCA disruption threshold is estimated to be $R_{n} \sim 2.0$ [31]. Complete discussion about the threshold for UCA destruction is given in the discussion section. This limit can be considered a limit below which UCA destruction will be at a minimum.

The normalized maximum radial oscillation $\left(R_{n}\right)$ as a function of frequency at varying acoustic pressures $\left(P_{\mathrm{A}}\right)$ for UCAs with $R_{0}$ of 1 and $4 \mu \mathrm{m}$ are shown in Fig. 1a, b, respectively. The resonance frequency of the UCA decreased and the radial oscillation increased with acoustic pressure. For the $1 \mu \mathrm{m}$ UCA, the resonance frequency decreased from the linear resonance frequency $\left(f_{\mathrm{r}}\right)$ of $8.21 \mathrm{MHz}$ at $1 \mathrm{kPa}$ to $5.46 \mathrm{MHz}\left(f_{\mathrm{s}}\right)$ at $340 \mathrm{kPa}$ acoustic pressure $\left(R_{n}=1.9\right)$. This corresponds to $33 \%$ decrease in the resonant frequency (Fig. 1a). For the $4 \mu \mathrm{m}$ UCA, the resonance frequency decreased from $1.21 \mathrm{MHz}\left(f_{r}\right)$ to $0.88 \mathrm{MHz}\left(f_{s}\right)$ at $105 \mathrm{kPa}\left(R_{n}=1.9\right)$ corresponding to $27 \%$ decrease in the resonant frequency (Fig. 1b).

The ratios of $f_{\mathrm{s}}$ to $f_{\mathrm{r}}\left(\frac{f_{\mathrm{s}}}{f_{\mathrm{r}}}\right)$ as a function of acoustic pressure for four UCA sizes $(1-4 \mu \mathrm{m})$ are shown in Fig. 2. The ratio $\frac{f_{\mathrm{s}}}{f_{\mathrm{r}}}$ decreased as the acoustic pressure increased. Moreover, the $\frac{f_{\mathrm{S}}}{f_{\mathrm{r}}}$ ratio decreased for increasing UCA size at a given acoustic pressure exposure. The rate of decrease of $\frac{f_{\mathrm{S}}}{f_{\mathrm{r}}}$ is approximately three times higher for UCA with $R_{0}=4 \mu \mathrm{m}$ compared to $R_{0}=1 \mu \mathrm{m}$. On average, the resonance frequency changed by $30-35 \%$ from the linear resonance frequency as the acoustic pressure increased. The larger UCAs reach the threshold of $R_{n}=1.9$ at lower pressures compared to smaller UCAs (105 vs $340 \mathrm{kPa}$ for the 4 and $1 \mu \mathrm{m} \mathrm{UCA}$, respectively). This is mainly due 

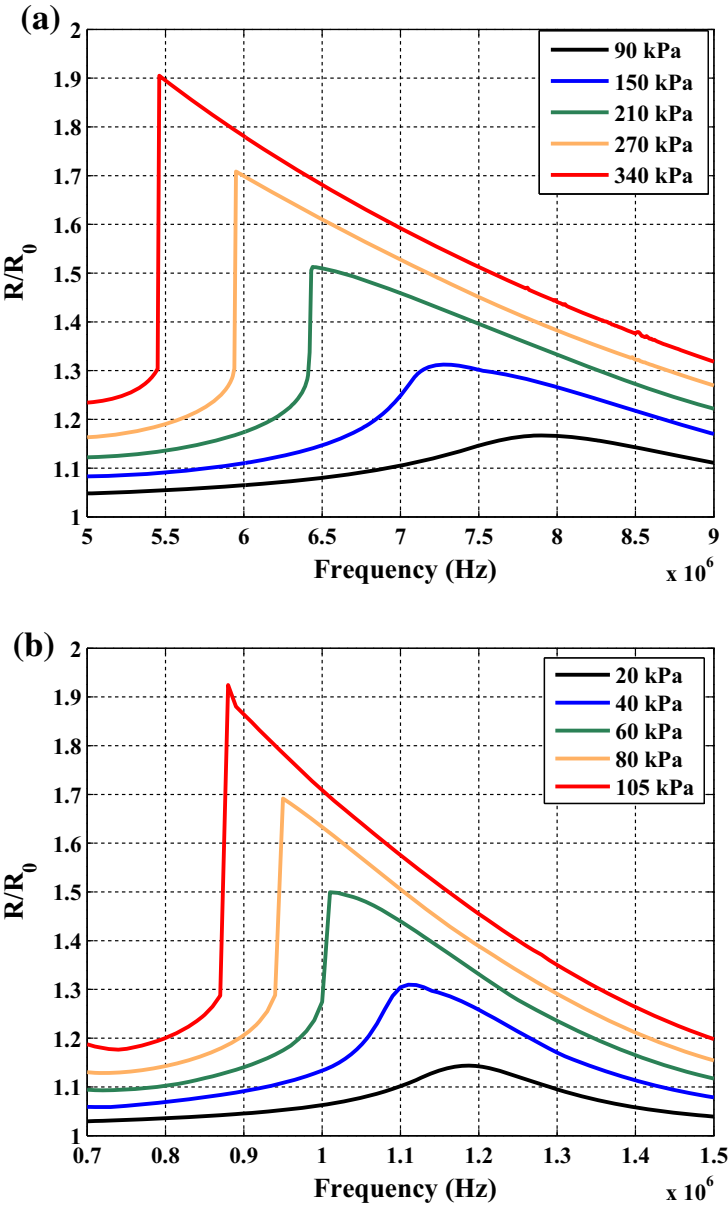

Fig. 1 Resonance curves at different driving pressures for a $R_{0}=1 \mu \mathrm{m}$ (linear resonance $\left.=8.21 \mathrm{MHz}\right), \mathbf{b} R_{0}=4 \mu \mathrm{m}$ (linear resonance $=1.21 \mathrm{MHz}$ ). The driving pressures used in the simulation are shown in the figure caption

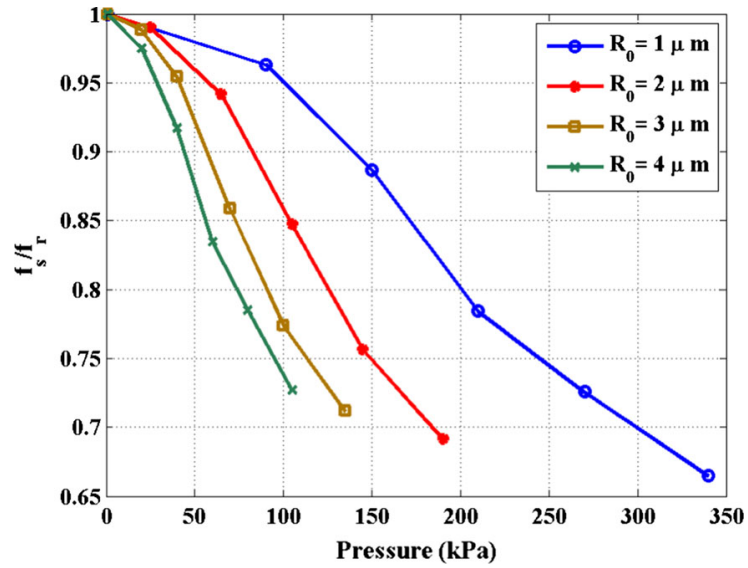

Fig. 2 Variation of the resonance frequencies versus acoustic pressure for UCAs with $R_{0}=1,2,3$ and $4 \mu \mathrm{m}$ to the larger effect of viscosity and surface tension for the smaller UCA oscillations [32].

\subsection{Bifurcation structure of the UCA driven with linear and pressure-dependent resonance frequencies}

The bifurcation structures of the radial oscillations of the 4 UCAs (representing 4 initial radii) were plotted as a function of $R_{n}$ versus the driving acoustic pressure $P_{\mathrm{A}}$. The driving frequencies were chosen firstly based on the linear resonance frequency and then the calculated pressure-dependent resonance frequencies for the appropriate ultrasound-driving pressure. The bifurcation analysis was performed over the 40 cycles of the 80 -cycle pulse to eliminate the transient behavior.

In order to investigate the effects of the shift in the resonance frequency on the behavior of the UCAs, the bifurcation diagrams of the UCAs were generated as a function of $R_{n}$ versus driving acoustic pressure $\left(P_{\mathrm{A}}\right)$. In each diagram, the driving frequency used was based on the pressure-dependent resonance frequency $\left(f_{\mathrm{s}}\right.$ in Fig. 1). The generated bifurcation diagrams were compared to the case of insonification with linear resonance frequency $\left(f=f_{\mathrm{r}}, P_{\mathrm{A}}=1 \mathrm{kPa}\right)$. The results of this comparison are shown in Fig. 3 for UCAs of $R_{0}=1$ (left column) and $4 \mu \mathrm{m}$ (right column) respectively.

The bifurcation diagrams of 1 and $4 \mu \mathrm{m}$ size UCAs with varying acoustic pressures $\left(P_{\mathrm{A}}\right)$ at their corresponding resonance frequencies $\left(f_{\mathrm{s}}\right)$ are shown in Fig. 3.

The bifurcation diagram for the $1 \mu \mathrm{m}$ UCA excited at its linear resonance frequency $\left(f_{\mathrm{r}}=8.21 \mathrm{MHz}\right)$ is shown in Fig. 3a. The UCA $(1 \mu \mathrm{m})$ undergoes perioddoubling bifurcation at $P_{\mathrm{A}}$ of $546 \mathrm{kPa}$ and $R_{n}$ of 1.54 , and exhibits $\frac{1}{2}$ subharmonics up to $661 \mathrm{kPa}\left(P_{\mathrm{A}}\right)$. With increasing pressure, the UCA shows period-doubling cascades and chaos at $P_{\mathrm{A}}$ of $700 \mathrm{kPa}$ and $R_{n}$ of 1.88 . The disruption threshold occurs at $790 \mathrm{kPa}$ pressure with $R_{n}=2$. According to Fig. 3a, the maximum $R_{n}$ which is obtained in the regime of period one is 1.54 (The red line indicates the destruction threshold).

A similar response is observed for the $4 \mu \mathrm{m}$ UCA at its linear resonance frequency $\left(f_{\mathrm{r}}=1.21 \mathrm{MHz}\right)$ (Fig. 3b). The $4 \mu \mathrm{m}$ UCA exhibits period-one oscillations with increasing amplitude up to $P_{\mathrm{A}}$ of $240 \mathrm{kPa}$ $\left(R_{n}=1.7\right)$, and above which the $4 \mu \mathrm{m}$ UCA undergoes successive period doubling $\left(P_{\mathrm{A}}=320 \mathrm{kPa}\right)$ to 
Fig. 3 Bifurcation diagram of the $\frac{R}{R_{0}}$ of the UCAs versus the driving acoustic pressure. In the left column, the UCA have an initial radius $R_{0}=1 \mu \mathrm{m}$, while in the right column, they have an initial radius of $4 \mu \mathrm{m}$, and are driven at the frequencies in the figure inset. The initial radius and excitation frequencies are:

a $R_{0}=1 \mu \mathrm{m}$

$f=8.21 \mathrm{MHz}$

b $R_{0}=4 \mu \mathrm{m}$

$f=1.21 \mathrm{MHz}$,

c $R_{0}=1 \mu \mathrm{m}$

$f=7.25 \mathrm{MHz}$,

d $R_{0}=4 \mu \mathrm{m}$

$f=1.11 \mathrm{MHz}$,

e $R_{0}=1 \mu \mathrm{m}$

$f=5.96 \mathrm{MHz}$,

f $R_{0}=4 \mu \mathrm{m}$

$f=0.95 \mathrm{MHz}$,

g $R_{0}=1 \mu \mathrm{m}$

$f=5.46 \mathrm{MHz}$ and

h $R_{0}=4 \mu \mathrm{m}$

$f=0.88 \mathrm{MHz}$. (Red line shows the destruction threshold). (Color figure online)
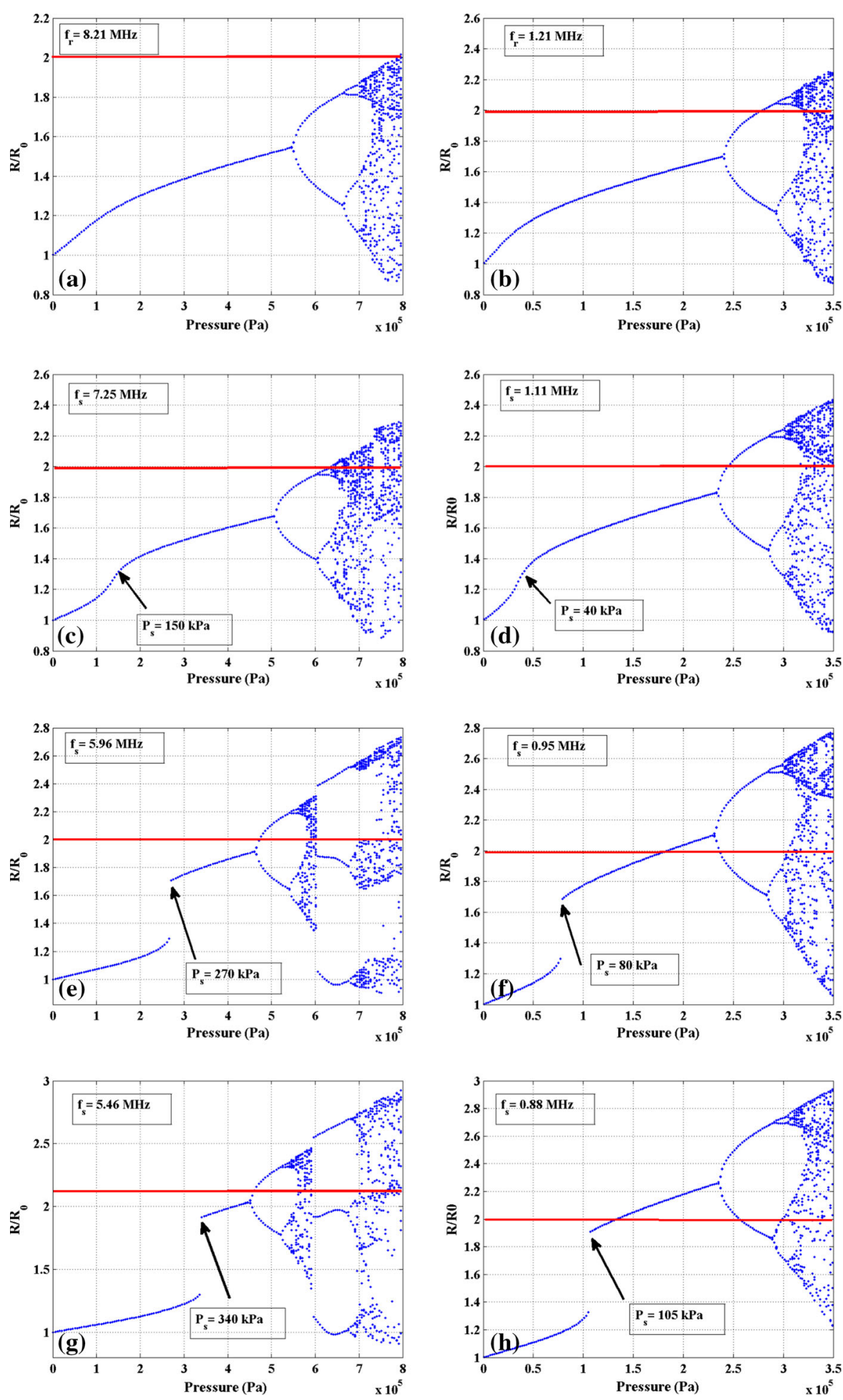
chaos. The destruction occurs at $280 \mathrm{kPa}$. As depicted in Fig. 3b, driving the UCA with $R_{0}=4 \mu \mathrm{m}$ with its linear resonance frequency results in a maximum period one $R_{n}$ of 1.7.

Figure $3 \mathrm{c}$ shows the bifurcation structure of the UCA with $R_{0}=1 \mu \mathrm{m}$ driven with $f=7.25 \mathrm{MHz}$ (the resonance frequency $f_{\mathrm{s}}$ at $P_{\mathrm{A}}=150 \mathrm{kPa}$, Fig. 1a). Compared to Fig. 3a, a change in the slope of increase in $R_{n}$ versus pressure is noticeable at $P_{\mathrm{A}}=150 \mathrm{kPa}$. The UCA driven with $f_{\mathrm{r}}$ exhibits a monotonic increase in $R_{n}$ as $P_{\mathrm{A}}$ is increased. As illustrated in Fig. 3b, for $P_{\mathrm{A}}<P_{\mathrm{S}}\left(P_{\mathrm{S}}=150 \mathrm{kPa}\right), R_{n}$ is smaller than the case depicted in Fig. 3a. After the $P_{\mathrm{A}}$ is increased above the $P_{\mathrm{S}}$, the $R_{n}$ undergoes a rapid growth and gets larger than the $R_{n}$ of that UCA driven with $f_{\mathrm{r}}$ (Fig. 3a). The oscillatory behavior follows a period-one regime with a higher $R_{n}$ compared to the case of the UCA driven with $f_{\mathrm{r}}$. Period-doubling occurs at $P_{\mathrm{A}}=506 \mathrm{kPa}$ while $R_{n}$ reaches $\sim 1.7$. Compared to the UCA driven with $f=f_{\mathrm{r}}, R_{n}$ reaches a higher value when the period doubling occurs. The UCA exhibits period two before the occurrence of further period-doubling cascades above $P_{\mathrm{A}}=606 \mathrm{kPa}$. The onset of chaos and destruction are concomitant at $P_{\mathrm{A}}=630 \mathrm{kPa}$ with $R_{n}=2$. Like the onset of period doubling, the pressure threshold for chaotic behavior is lower compared with the case of $f=f_{\mathrm{r}}$.

Figure $3 \mathrm{~d}$ shows the bifurcation structure of the UCA with $R_{0}=4 \mu \mathrm{m}$ driven with $f=1.11 \mathrm{MHz}$ (the resonance frequency $f_{\mathrm{s}}$ at $P_{\mathrm{A}}=40 \mathrm{kPa}$, Fig. 1a). The dynamics are very similar to the UCA in Fig. 3c. The oscillation amplitude is below the oscillation amplitude of the UCA sonicated with $f=f_{\mathrm{r}}$ but only for $P_{\mathrm{A}}<P_{\mathrm{S}}=40 \mathrm{kPa}$. For acoustic pressure amplitudes greater than $P_{\mathrm{S}}$, the oscillations undergo a significant increase in amplitude and in a manner similar to that presented in Fig. 3b. For these conditions, the destruction of the UCA with $R_{0}=4 \mu \mathrm{m}$ occurs at $247 \mathrm{kPa}$.

Figure $3 \mathrm{e}$ depicts the bifurcation diagram of the UCA with $R_{0}=1 \mu \mathrm{m}$ when driven with $f=$ $5.96 \mathrm{MHz}\left(f_{\mathrm{s}}\right.$ at $\left.P_{\mathrm{A}}=270 \mathrm{kPa}\right)$. Compared to Fig. 3a, $R_{n}$ is lower for $P_{\mathrm{A}}<P_{\mathrm{S}}=270 \mathrm{kPa}$. Once the $P_{\mathrm{A}}$ is increased above $270 \mathrm{kPa}, R_{n}$ undergoes a rapid growth and exhibits a saddle-node bifurcation to a higher oscillation amplitude $\left(R_{n} \sim 1.71\right)$, which is $23 \%$ larger than the $R_{n}$ in Fig. 3 a (at the same $P_{\mathrm{A}}$ ). The UCA continues period one oscillations at a higher $R_{n}$ compared to Fig. 3a, c and undergoes period doubling at $P_{\mathrm{A}} \sim 461 \mathrm{kPa}$ at which point the $R_{n}$ reaches
1.91. This is $24 \%$ larger than the maximum period one $R_{n}$ in Fig. 3a. The chaotic behavior appears at $P_{\mathrm{A}}=577 \mathrm{kPa}$ with $R_{n} 2.20$. The threshold $P_{\mathrm{A}}$ for the onset of period doubling, destruction (at $475 \mathrm{kPa}$ ), and chaotic oscillations is lower than both the threshold $P_{\mathrm{A}}$ for $f_{\mathrm{r}}=8.21 \mathrm{MHz}$ and $f_{\mathrm{s}}=7.25 \mathrm{MHz}$.

Figure $3 f$ illustrates the bifurcation diagram of the UCA with $R_{0}=4 \mu \mathrm{m}$ when driven with $f=$ $0.95 \mathrm{MHz}\left(f_{\mathrm{s}}\right.$ at $\left.P_{\mathrm{A}}=80 \mathrm{kPa}\right)$. Compared to Fig. 3b, $R_{n}$ is lower for a $P_{\mathrm{A}}<P_{\mathrm{S}}=80 \mathrm{kPa}$. Once the $P_{\mathrm{A}}$ is increased above $P_{\mathrm{s}}=60 \mathrm{kPa}, R_{n}$ undergoes a rapid growth and exhibits a saddle-node bifurcation to a higher oscillation amplitude $\left(R_{n} \sim 1.68\right)$, which is $26 \%$ larger than the $R_{n}$ in Fig. $3 \mathrm{~b}$ at the same $P_{\mathrm{A}}$. The UCA continues period one oscillations at a higher $R_{n}$ compared with Fig. 3b, d. The radial oscillations pass the destruction threshold at $183 \mathrm{kPa}$. The UCA undergoes period doubling at $P_{\mathrm{A}} 231 \mathrm{kPa}$ while $R_{n}$ reaches $\sim 2$.2. The maximum period-one oscillations in this case is $17 \%$ larger than the maximum period one $R_{n}$ in Fig. 3b. The chaotic behavior appears at $P_{\mathrm{A}}=300 \mathrm{kPa}$ with $R_{n} \sim 2.6$. The threshold $P_{\mathrm{A}}$ for the onset of period doubling and chaotic oscillations is lower than both the $f_{\mathrm{r}}=1.21 \mathrm{MHz}$ and $f_{\mathrm{s}}=1.11 \mathrm{MHz}$. There is a high degree of similarity in the dynamics between Fig. 3f, e. This indicates a general behavior for the UCAs sonicated with their pressure-dependent resonance frequency.

The bifurcation diagram of the UCA with $R_{0}=$ $1 \mu \mathrm{m}$ sonicated with $f=5.46 \mathrm{MHz}$ (the $f_{\mathrm{s}}$ at $P_{\mathrm{A}}=$ $340 \mathrm{kPa}$ ) is presented in Fig. $3 \mathrm{~g}$. As shown, the $R_{n}$ is less than the previous cases (Fig. 3a, c, e) for $P_{\mathrm{A}}<$ $P_{\mathrm{S}}=340 \mathrm{kPa}$. However, once the $P_{\mathrm{A}}$ is increased above $340 \mathrm{kPa}, R_{n}$ exhibits a significant growth and becomes larger than the $R_{n}$ in Fig. (3a, c, e). At this $P_{\mathrm{A}}$ amplitude, the $R_{n}$ is $35 \%$ larger than when driven with $f_{\mathrm{r}}$. The oscillation is of period one for $P_{\mathrm{A}}<451 \mathrm{kPa}$ with a larger $R_{n}$ (in $330<P_{\mathrm{A}}<451 \mathrm{kPa}$ ) compared to the previous cases. The radial oscillations of the UCA pass the destruction threshold at $416 \mathrm{kPa}$. The UCA undergoes period doubling at $451 \mathrm{kPa}$ and $R_{n} \sim 2.04$. Finally, the oscillations become chaotic after a series of successive period-doubling bifurcations at $\sim 546 \mathrm{kPa}$. In this case, a full nondestructive amplitude $\left(R_{n}=2\right)$ of period one is developed before the UCA undergoes period doubling.

Figure $3 \mathrm{~h}$ depicts the bifurcation diagram of the UCA with $R_{0}=4 \mu \mathrm{m}$ insonified with $f=0.88 \mathrm{MHz}$ (the $f_{\mathrm{s}}$ at $P_{\mathrm{A}}=105 \mathrm{kPa}$ ). As shown, the $R_{n}$ is less than 
Fig. 4 Backscattered pressure amplitude as a function of pressure for linear and pressure-dependent resonance frequencies when a $R_{0}=1 \mu \mathrm{m}, \mathbf{b}$ $R_{0}=2 \mu \mathrm{m}, \mathbf{c} R_{0}=3 \mu \mathrm{m}$ and $\mathbf{d} R_{0}=4 \mu \mathrm{m}$. The pressure-dependent resonance frequencies are in the figure legends
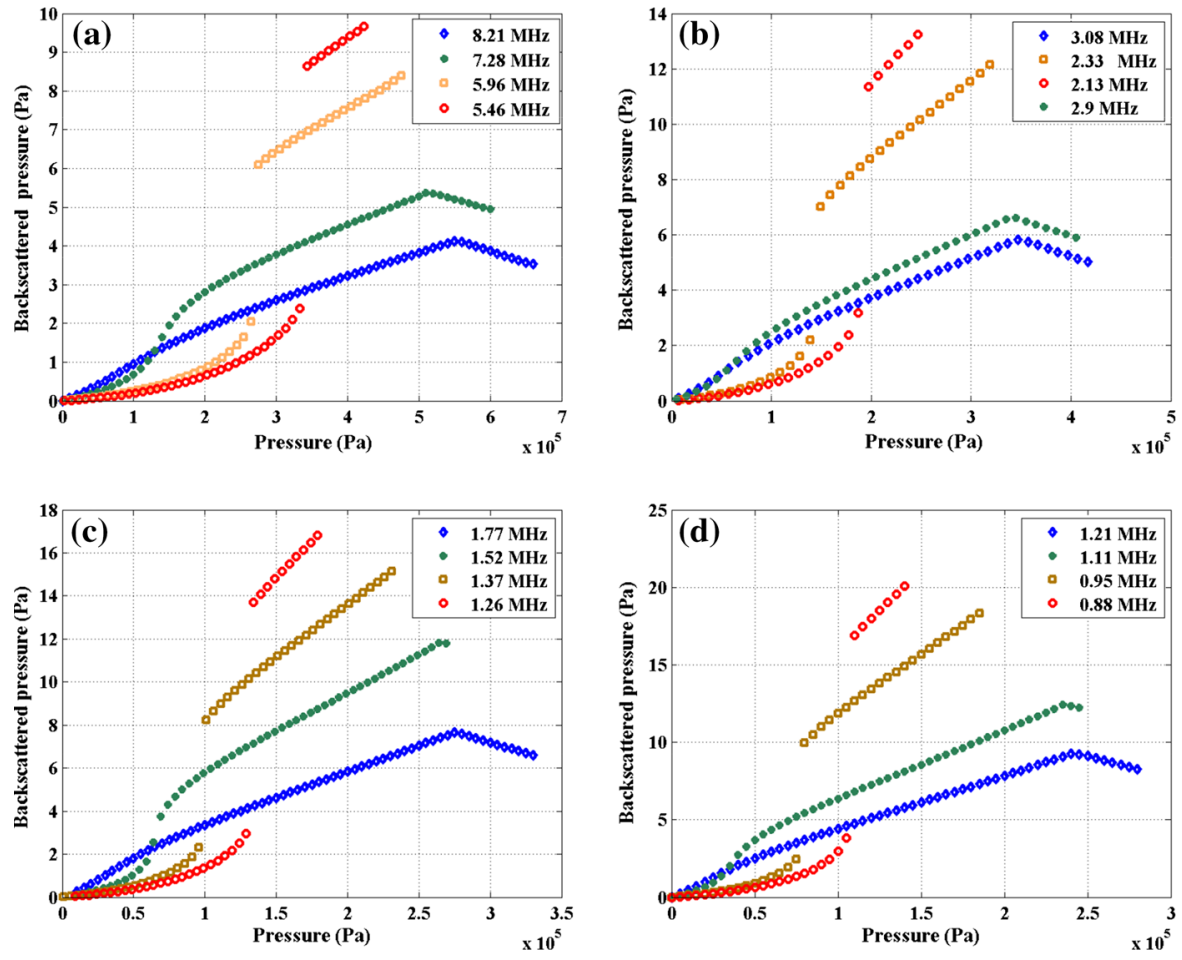

the previous cases (Fig. 3b, d, f) for $P_{\mathrm{A}}<P_{\mathrm{s}}=105 \mathrm{kPa}$. However, once the $P_{\mathrm{A}}$ is increased above $105 \mathrm{kPa}, R_{n}$ exhibits a significant growth and becomes larger than the $R_{n}$ in Fig. (3a, d, f). At $P_{\mathrm{A}}=P_{\mathrm{S}}=105 \mathrm{kPa}$ amplitude, the $R_{n}$ is $\sim 30 \%$ larger than when driven with $f_{\mathrm{r}}$. The oscillation is of period one for $P_{\mathrm{A}}<231 \mathrm{kPa}$ with a larger $R_{n}$ (in $105<P_{\mathrm{A}}<231 \mathrm{kPa}$ ) compared to the previous cases. The destruction threshold occurs at $137 \mathrm{kPa}$. The UCA undergoes period doubling at $232 \mathrm{kPa}\left(R_{n} \sim 2.32\right)$. Finally, the oscillations become chaotic after a series of successive perioddoubling bifurcations at $310 \mathrm{kPa}$. Again, the similarity between Fig. $3 \mathrm{~g}$, $\mathrm{h}$ is noticeable indicating a general trend in the UCA behavior sonicated with pressuredependent resonance. In both cases, a full-amplitude period-one oscillation is achieved. The importance of the full-amplitude period-one oscillations are in the backscattered pressure and will be discussed in the next section.

\subsection{Maximum backscattered pressure in the regime of stable UCA oscillations}

The backscattered pressure amplitude $\left(P_{\mathrm{sc}}\right)$ was numerically calculated using Eq. 3 for all of the exposure parameters. In each case, the excitation pressure range was chosen so that the $R_{n}$ remained below the UCA destruction threshold $\left(R_{n}=2\right)$. This range was chosen by examination of the bifurcation diagrams and ensuring that $\frac{R_{\max }}{R_{0}}<2$ (red line in Fig. 3). The results are shown in Fig. 4a-d for UCAs with $R_{0}=1,2,3$ and $4 \mu \mathrm{m}$.

The backscattered pressure amplitude $\left(P_{\mathrm{Sc}}\right)$ from UCAs of different sizes $(1-4 \mu \mathrm{m})$ at varying resonance frequencies is shown in Fig. 4. The $P_{\text {sc }}$ increases with acoustic pressure for all UCA sizes and acoustic frequencies. At low acoustic pressures, a higher $P_{\mathrm{sc}}$ is achieved at the UCAs linear resonance frequency $\left(f_{\mathrm{r}}\right)$. However, with increasing acoustic pressure and at pressure-dependent resonance frequency, the $P_{\mathrm{sc}}$ amplitude significantly increases.

As shown in Fig. 4a-d, driving the UCA with its linear resonance frequency $\left(f_{\mathrm{r}}\right)$ results in a higher backscattered pressure amplitude $\left(P_{\mathrm{sc}}\right)$, but only at low pressures of insonification. When sonicated with its linear resonance frequency, the $P_{\mathrm{sc}}$ increases linearly alongside the driving acoustic pressure until the UCA undergoes the period-doubling bifurcation. The occurrence of the period-doubling bifurcation is concomitant with a decrease in the $P_{\mathrm{sc}}$ although the UCA displays 
a higher $R_{n}$. This will be explained in the next section.

Figure 4a-d illustrate that when a UCA is sonicated with its pressure-dependent resonance frequency $\left(f_{\mathrm{s}}\right)$, the diagram has two distinct regions, $P_{\mathrm{A}}<P_{\mathrm{S}}$ and $P_{\mathrm{A}}>P_{\mathrm{s}}$. For $P_{\mathrm{A}}<P_{\mathrm{s}}$ the $P_{\mathrm{sc}}$ is less than the backscattered pressure amplitude of a UCA driven with $f_{\mathrm{r}}$. However, as soon as the pressure is increased above $P_{\mathrm{s}}$, the $P_{\mathrm{sc}}$ increases significantly becoming much larger than the $P_{\mathrm{sc}}$ of a UCA driven with its linear resonance frequency $f_{\mathrm{r}}$. For example in Fig. $4 \mathrm{a}$, the $P_{\mathrm{sc}}$ of a $R_{0}=1 \mu \mathrm{m}$ UCA driven with $f_{\mathrm{s}}=5.46 \mathrm{MHz}$ $\left(P_{\mathrm{s}}=340 \mathrm{kPa}\right)$ becomes $\sim$ threefold larger than the $P_{\mathrm{sc}}$ of the same UCA driven with $f_{\mathrm{r}}=8.21 \mathrm{MHz}$ as soon as the pressure increases above $340 \mathrm{kPa}$.

A comparison between Figs. 3 and 4 shows that the sudden increase in $P_{s c}$ is concomitant with the saddlenode bifurcation in $R_{n}-P_{\mathrm{A}}$ bifurcation diagrams. The increase is more significant for higher $P_{\mathrm{s}}$ and lower $f_{\mathrm{s}}$. In this regard, if $f_{\mathrm{s} 1}, f_{\mathrm{s} 2}$ and $f_{\mathrm{s} 3}$ are the pressuredependent resonance frequencies of the UCA at the acoustic pressures of $P_{\mathrm{s} 1}, P_{\mathrm{s} 2}$ and $P_{\mathrm{s} 3}$ where $P_{\mathrm{s} 1}<$ $P_{\mathrm{s} 2}<P_{\mathrm{s} 3}$; the $P_{\mathrm{sc}}$ follows the order of $P_{\mathrm{sc} 3}>P_{\mathrm{sc} 2}>$ $P_{\mathrm{sc} 1}$ as soon as $P_{\mathrm{A}}$ increases above $P_{\mathrm{s} 3}$.

Figure 4 contains very important information regarding the maximum possible backscatter from an UCA $\left(P_{\text {scmax }}\right)$. Driving the UCA with its linear resonance frequency does not result in the maximum backscatter. When the pressure-dependent resonance frequency is used and the pressure is increased above $P_{\mathrm{s}}$, the $P_{\text {sc }}$ increases significantly. The backscattered pressure amplitude continues to increase alongside pressure until the UCA is destroyed $\left(R_{n}>2\right)$. Just below this critical pressure for UCA destruction, the maximum possible backscattered pressure $\left(P_{\mathrm{scmax}}\right)$ is at its highest for the UCA driven with $f_{\mathrm{s}}$.

For example, in Fig. 4a, the $P_{\text {scmax }}$ for $R_{0}=1 \mu \mathrm{m}$ driven with $f_{\mathrm{r}}=8.15 \mathrm{MHz}$ is $4 \mathrm{~Pa}$ and occurs at $P_{\mathrm{A}}=$ $542 \mathrm{kPa}$. However, when the same UCA is driven with $f_{\mathrm{s}}=5.4 \mathrm{MHz}$ the $P_{\mathrm{scmax}}$ reaches $9.3 \mathrm{~Pa}$ and happens at $P_{\mathrm{A}}=408 \mathrm{kPa}$. It should be noted that not only is the backscatter pressure maximized, but also it is occurring at a lower driving pressure. This can have significant advantages in increasing the signal to noise ratio (SNR) and contrast to tissue ratio (CTR) in medical imaging applications. This is because the SNR decreases for linear scatterers (such as tissues) at lower sonication pressures while at the same time the $P_{\mathrm{sc}}$ from the UCA increases at these lower excitation pressures.

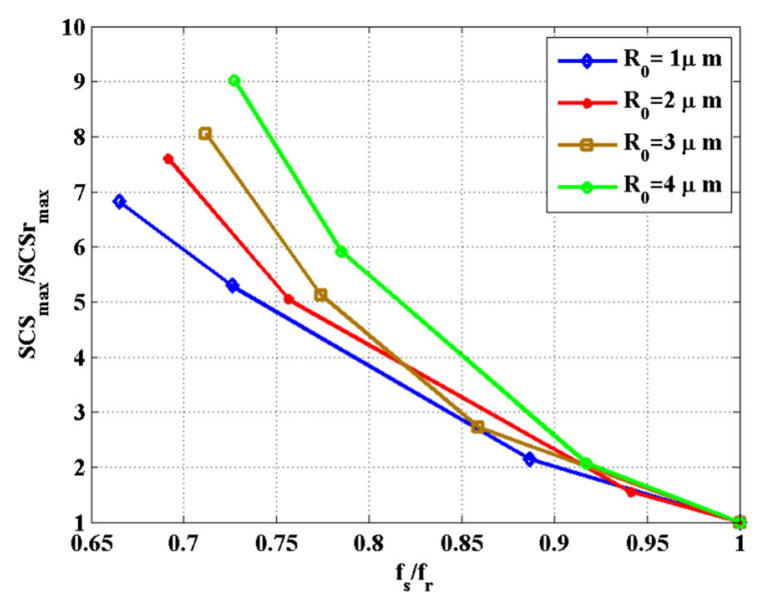

Fig. 5 The normalized maximum possible scattering cross section while avoiding the UCA destruction as a function of $\frac{f_{\mathrm{s}}}{f_{\mathrm{r}}}$. In each case, the maximum possible scattering cross section $\left(\mathrm{SCS}_{\max }\right)$ is normalized by the maximum possible scattering cross section for a UCA driven with its linear resonance frequency $\left(\mathrm{SCSr}_{\max }\right)$

In summary, it is observed that in the region of nondestructive oscillations $\left(R_{n}<2\right)$, the maximum possible backscattered pressure has an upper limit when the UCA is driven with $f_{\mathrm{r}}$. This limit can significantly be enhanced through optimizing the driving frequency in terms of the pressure-dependent resonant frequency $f_{\mathrm{s}}$ and applying an acoustic pressure that is greater than a threshold $P_{\mathrm{s}}$. The maximum possible nondestructive backscattered pressure occurs in the region of the period-one oscillations. Occurrence of the period doubling is concomitant with a decrease in the backscattered pressure.

\subsection{Comparison between the maximum possible backscatter cross section}

In order to investigate the effect of the pressuredependent resonance on the scattering properties of the UCAs, the maximum possible scattering cross section $\left(\mathrm{SCS}_{\max }\right)$ was calculted using Eq. 4. The maximum possible backscattered pressure derived in Fig. 4 was used in the calculation of the $\mathrm{SCS}_{\max }$. These values were then normalized by the maximum possible SCS in the case of excitation with the linear resonance frequency $\left(\mathrm{SCSr}_{\max }\right)$. These values were plotted as a function of $\frac{f_{\mathrm{s}}}{f_{\mathrm{r}}}$ in Fig. 5.

As it is illustrated in Fig. 5, driving the UCA with $f_{\mathrm{s}}$ results in a larger scattering cross section compared 
Fig. 6 Comparison between the period-one and period-two behaviors of the UCA with $R_{0}=1 \mu \mathrm{m}$ driven with $8.21 \mathrm{MHz}$ of frequency. In left column $P_{\mathrm{A}}=533 \mathrm{kPa}:$ a radial oscillations, b velocity, c acceleration, $\mathbf{d}$ backscattered pressure. In right column $P_{\mathrm{A}}=640 \mathrm{kPa}$ : e radial oscillations, $\mathbf{f}$ velocity, $\mathbf{g}$ acceleration, and h backscattered pressure
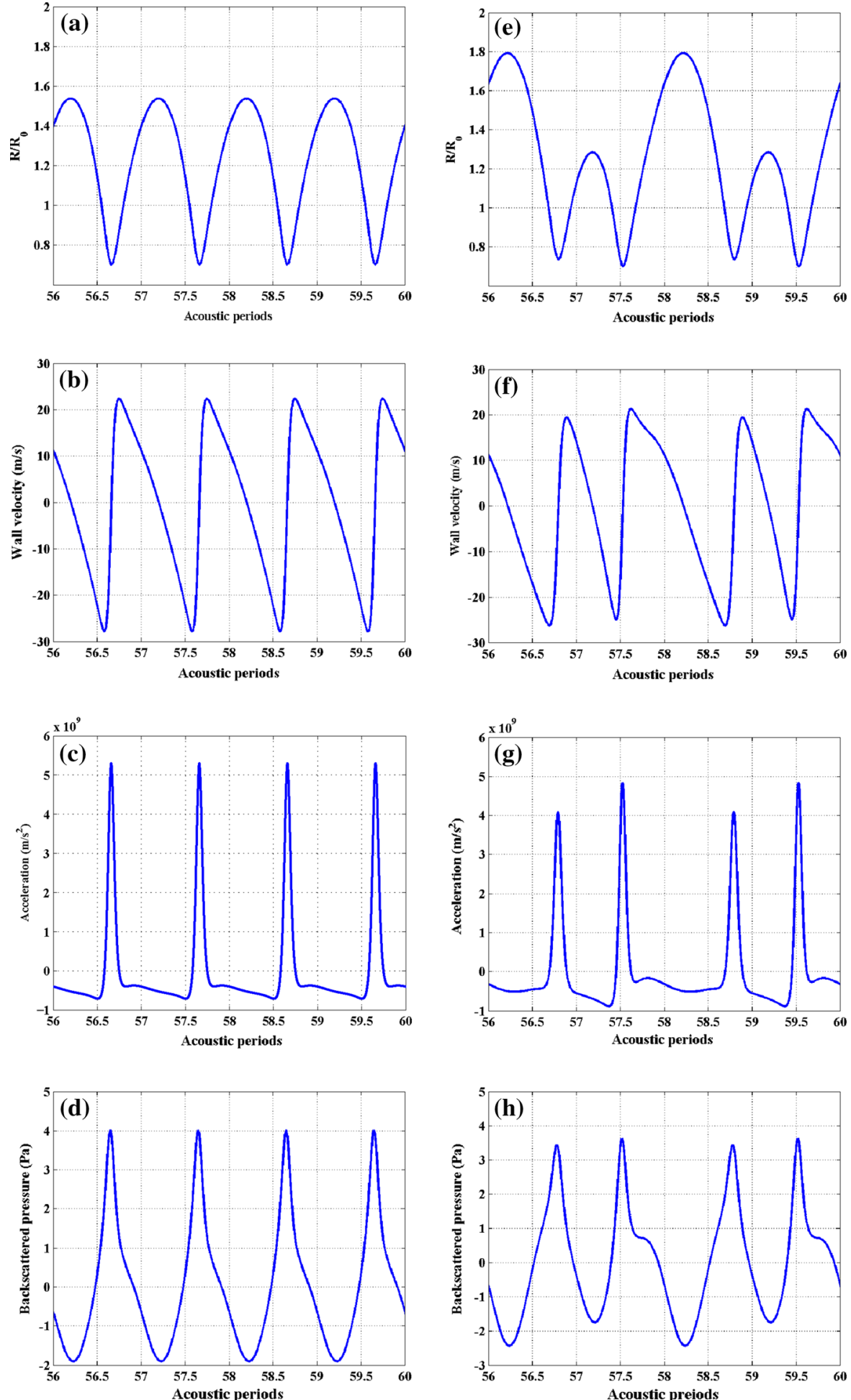
with excitation with $f_{\mathrm{r}}$. The $\mathrm{SCS}_{\max }$ increases as $f_{\mathrm{s}}$ decreases. As an example, exciting an $R_{0}=4 \mu \mathrm{mUCA}$ with $f_{\mathrm{s}}=0.86 \mathrm{MHz}$ results in a $\mathrm{SCS}_{\max }$ which is nine times greater than the $\mathrm{SCS}_{\max }$ of $f_{\mathrm{r}}=1.21 \mathrm{MHz}$. It can also be concluded from Fig. 5 that the $\mathrm{SCS}_{\max }$ increases faster versus $\frac{f_{\mathrm{s}}}{f_{\mathrm{r}}}$ for the larger UCAs.

\subsection{The concomitant decrease in maximum backscattered pressure with period doubling}

In Sect. 3.3, it was shown that the backscattered pressure decreases when the period doubling occurs. At the first glance, this appears counterintuitive as the decrease occurs for a higher $R_{n}$. After period doubling, while pressure increases, $R_{n}$ keeps increasing but the backscattered pressure decreases. Comparing the radial oscillations between the period-one regime and the period-two regime (after the period doubling has taken place) shows that when period doubling occurs, the magnitude of the UCA contraction decreases, and thus, the UCA velocity and acceleration during the contraction phase of the UCA oscillation are smaller compared with the period-one oscillations prior to period doubling.

This phenomenon is shown in detail in Fig. 6a-d. Figure 6a shows the radial oscillations of the UCA with $R_{0}=1 \mu \mathrm{m}$ driven with $f_{\mathrm{r}}=8.21 \mathrm{MHz}$ and acoustic pressure of $533 \mathrm{kPa}$ as a function of the driving acoustic periods. This corresponds to a UCA undergoing periodone oscillations before the occurrence of the period doubling (Fig. 3a). The corresponding wall velocity, acceleration, and backscattered pressure are shown in Fig. 6b-d. Figure 6e illustrates the radial oscillations of the same UCA when driven with an acoustic pressure of $640 \mathrm{kPa}$. According to Fig. 3a, this condition corresponds for period-two oscillations after the perioddoubling bifurcation. The corresponding wall velocity, acceleration and backscattered pressure are shown in Fig. 6f-h. It can be observed in Fig. 6e that despite the increasing UCA radial oscillation amplitude, the magnitude of the contraction is smaller than in Fig. 6a. This results in a smaller velocity and acceleration for the UCA as shown in Fig. $6 f-g$. As a consequence, the corresponding backscattered pressure is larger for the UCA undergoing period-one oscillation (Fig. 6d).

The concomitant decrease in the $P_{\mathrm{sc}}$ amplitude with period doubling was only shown for the frequency range that was studied in this paper. The decrease in
$P_{\mathrm{sc}}$ was shown only for UCAs being sonicated with their resonance and pressure-dependent resonance frequencies. The frequencies which are considered in our study are between the resonance frequency $\left(f_{\mathrm{r}}\right)$ and the pressure-dependent resonance frequency $\left(f_{\mathrm{s}}\right)$ down to $0.665 \times f_{\mathrm{r}}$. It should be noted that in different cases like sonication of the UCA with its second harmonic resonance frequency $\left(0.5 \times f_{\mathrm{r}}\right)$ or sonication with the subharmonic resonance frequency $\sim 2 \times f_{\mathrm{r}}$ the behavior of the UCA will be different and one may not see the same decrease in the backscattered pressure concomitant with period doubling. However, as the main purpose of this study is the understanding of the dynamics of the resonant UCAs, we have not studied the above-mentioned frequency ranges. Investigation of the behavior of the harmonically or subharmonically resonant UCAs are beyond the scope of this paper.

\section{Effect of the initial condition on the resonance curves and bifurcation structure of the UCAs}

As it was discussed earlier, to generate the resonance curves and the bifurcation diagrams, the ICs were chosen to be $R(t=0)=R_{0}$ and $\dot{R}(t=0)=0$. However, the dynamics of a nonlinear system like UCA is heavily dependent on the initial conditions. The effect of random ICs on the dynamics and resonance curves of bubble oscillators have been investigated in $[1,7,30]$. The results of these studies have shown that there can be coexisting stable solutions for a given parameter range. In order to visualize all the solutions for a given parameter range, the effect of random ICs should be studied. In order to complete the analysis on the dynamics of the UCAs driven with their pressuredependent resonance frequency and test the effect of the IC on the solutions of the system in this regime, we have calculated one resonance curve and one bifurcation diagram considering ten random ICs [30] at each step.

Figure 7 shows the resonance frequency of a UCA with $R_{0}=1 \mu \mathrm{m}$, sonicated with an ultrasound pulse whose pressure amplitude is $P_{\mathrm{A}}=264 \mathrm{kPa}$. At each frequency step, the radial dynamics of the UCA is simulated for 10 random initial conditions (ICs) and the $\frac{R_{\max }}{R_{0}}$ is plotted for every IC. Compared to the case of sonication with ICs of $R(t=0)=R_{0}$, the resonance frequency may occur at a lower frequency 
Fig. 7 The pressure-dependent resonance frequency of the UCA with $R_{0}=1 \mu \mathrm{m}$ at $P_{\mathrm{A}}=264 \mathrm{kPa}$ with ten random ICs (blue superimposed) and when the ICs are $R(t=0)=R_{0}$ and $\dot{R}(t=0)=0$ (red). (Color figure online)

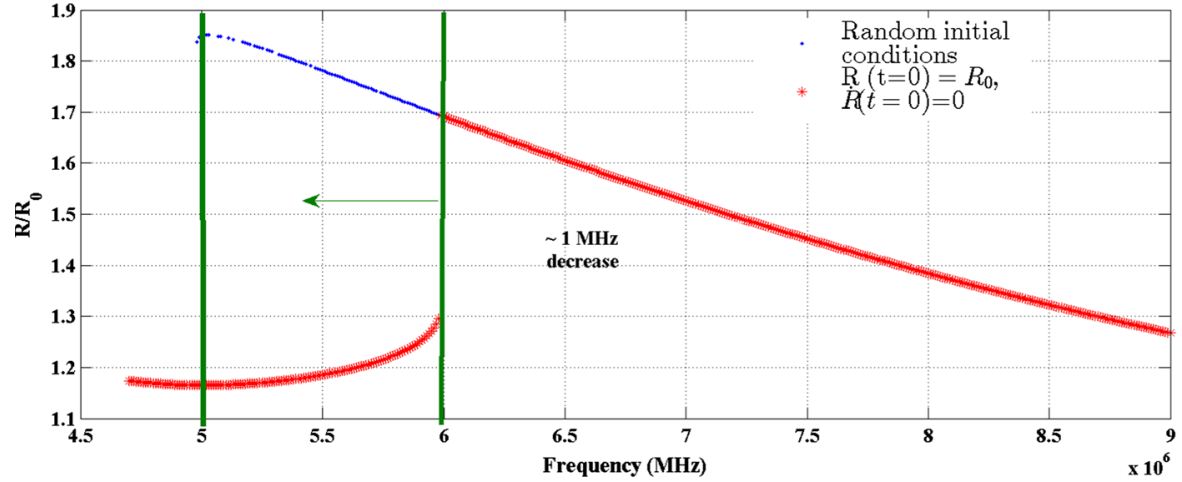

Fig. 8 The bifurcation diagram of the UCA with $R_{0}=1 \mu \mathrm{m}$ sonicated with $f=6 \mathrm{MHz}$ with ten random initial conditions (blue superimposed) and when the initial conditions are $R(t=0)=R_{0}($ red $)$. (Color figure online)

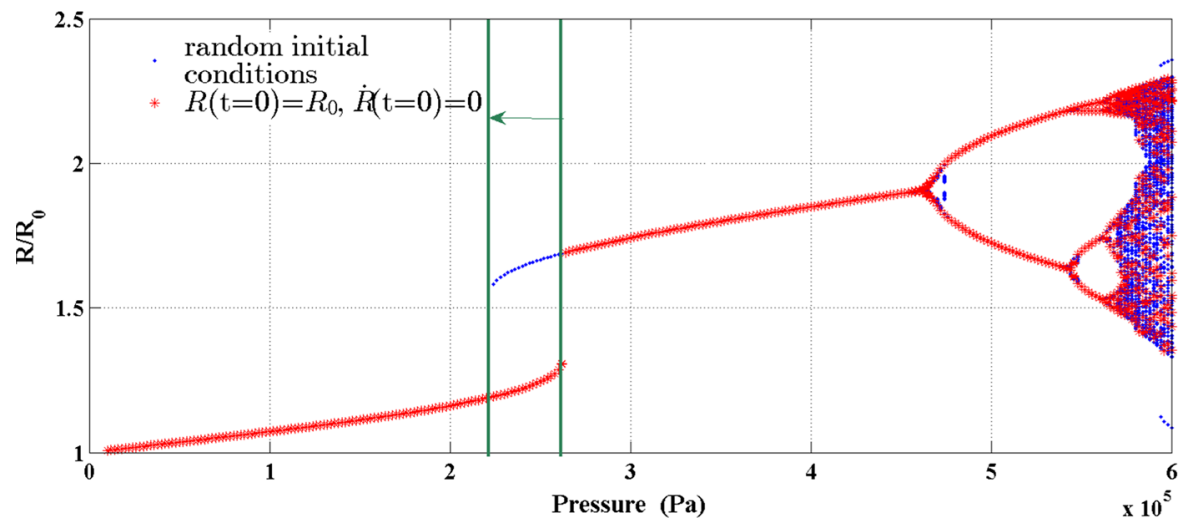

( $\sim 1 \mathrm{MHz}$ lower) with a higher amplitude. Also note the coexisting attractors for the frequency range of $\sim 5-6 \mathrm{MHz}$.

Figure 8 shows the bifurcation diagrams of the UCA with $R_{0}=1 \mu \mathrm{m}$ for two different set of ICs. The blue curve shows the bifurcation diagram for the IC of $R(t=$ $0)=R_{0}$ and $\dot{R}(t=0)=0$, while the red curve shows the bifurcation diagram for ten random IC at each pressure step. The sonication frequency is $6 \mathrm{MHz}$ which is the pressure-dependent resonance frequency of the $\operatorname{UCA}\left(f_{\mathrm{s}}\right)$ at $P_{\mathrm{A}}=264 \mathrm{kPa}$ for $R(t=0)=R_{0}$ and $\dot{R}(t=0)=0$ calculated in Fig. 7. As it is illustrated, the UCA starting with $R(t=0)=R_{0}$ and $\dot{R}(t=$ $0)=0$ (red), starts with period-one oscillations which undergo a saddle-node bifurcation at $P_{\mathrm{A}}=264 \mathrm{kPa}$ to a higher amplitude. However, the UCA starting with the random ICs, exhibit a slight different behavior. There is coexisting period-one oscillations in for $224 \mathrm{kPa} \leq$ $P_{\mathrm{A}}<264 \mathrm{kPa}$. Depending on the IC, the UCA exhibit the saddle-node bifurcation to a higher amplitude for a lower $P_{\mathrm{A}}$.

\section{Discussion and conclusion}

The resonance frequency of the UCAs decreases with increasing acoustic pressure. It was shown that the shift in the resonance significantly influences the oscillatory behavior of the UCA. The key findings can be summarized as follows:

When the bifurcation diagrams of the $\left(R_{n}=\frac{R}{R_{0}}\right)$ versus acoustic pressure $\left(P_{\mathrm{A}}\right)$ of an UCA driven with its linear resonance frequency $\left(f_{\mathrm{r}}\right)$ are compared to the case of insonification with $f_{\mathrm{s}}$ (resonance frequency at the acoustic pressure $P_{\mathrm{S}}$ ), it is seen that $R_{n}$ undergoes either a steep rise or a saddle-node bifurcation at the excitation pressure $P_{\mathrm{s}}$. After a saddle-node bifurcation, the UCA continues oscillations in the same way as the UCA driven with $f_{\mathrm{r}}$ but with a higher $R_{n}$. Both UCAs undergo a period-doubling transition to chaos; however, the UCAs driven with $f_{\mathrm{s}}$ exhibit period doubling and chaotic oscillations at lower pressure thresholds.

When the $R_{n}-P_{\mathrm{A}}$ bifurcation diagrams are compared for UCAs driven with $f_{\mathrm{s} 1}$ and $f_{\mathrm{s} 2}$ (nonlinear res- 
onance frequencies corresponding to $P_{\mathrm{s} 1}$ and $P_{\mathrm{s} 2}$ with $\left.P_{\mathrm{s} 2}>P_{\mathrm{s} 1}\right)$, both UCAs exhibit the saddle-node bifurcation with the difference of a saddle-node bifurcation to a higher amplitude in case of the UCA driven with $f_{\mathrm{s} 2}$. In addition, the UCA destruction $\left(R_{n}>2\right)$ and the transition to chaos occur at a lower pressure threshold for the UCA driven with $f_{\mathrm{s} 2}$. When the backscattered pressures are compared, it was shown that the acoustic pressure range can be divided into two distinct regions $\left(P_{\mathrm{A}}<P_{\mathrm{S}}\right.$ and $\left.P_{\mathrm{A}}>P_{\mathrm{S}}\right)$. For $P_{\mathrm{A}}<P_{\mathrm{s}}$, the backscattered pressure is higher for the UCA driven with $f_{\mathrm{r}}$. However, for the UCA driven with $f_{\mathrm{s}}$, while $P_{\mathrm{A}}>P_{\mathrm{S}}$ the backscattered pressure undergoes a significant increase and is greater than for the case of the excitation with $f=f_{\mathrm{r}}$. This is true for all of the applied driving acoustic pressure values for which $P_{\mathrm{A}}>P_{\mathrm{s}}$. The main advantage of sonication with $f_{\mathrm{s}}$ is the increased backscatter from UCAs at lower driving acoustic pressures. This can result in an enhanced SNR and CTR in a clinical imaging setting.

The threshold for destruction was set based on the criteria which was formulated by Flynn [33] and later was used in [31]. According to the formulation by FLynn, when $\frac{R_{\max }}{R_{0}}>2$, inertial forces dominate the collapse of the UCA and increasing quantities of kinetic energy will be transferred to the collapsing bubble as the surrounding liquid converges, while at the same time, decreasing amount of this energy is lost as a result of dissipation. Transient collapse is defined as a phenomenon where significant amount of energy is concentrated in the bubble, and the effect of the energy supply outweighs the energy dissipation [34]. So the destruction criterion by Flynn is set as $\frac{R_{\max }}{R_{0}}>2$.

There is also another criterion based on the ratio of $\frac{R_{\max }}{R_{0}}$ which is developed by Apfel [35]. In this criterion, transient collapse occurs when $\frac{R_{\max }}{R_{0}}$ is between 2 and 2.3. In this work, we have used the Flynn's criterion to ensure there is no transient collapse, as it predicts the minimum expansion ratio for bubble destruction.

There have been a number of experimental studies on the destruction of the ultrasound contrast agents. These studies employed mainly two techniques: (1) fast optical measurements of the UCA radius [36-39], (2) Acoustical method by analyzing the post-excitation signal after collapse [40-42]. Results of the large number of optical measurements of the UCA oscillations in [36-39] have shown that although some UCAs may undergo large amplitude oscillations and are not destroyed when $\frac{R_{\max }}{R_{0}}>2$, there is no or very little evidence of UCA destruction when $\frac{R_{\max }}{R_{0}}<2$. In other words, the majority of the UCAs are destroyed once $\frac{R_{\max }}{R_{0}}>2$ although some few UCAs may exhibit higher expansion ratios. In another series of acoustical investigations on the UCA destruction, the post-excitation signal by UCA has been used as an indicator for UCA destruction [40-42]. The results of these studies illustrated no evidence of UCA destruction when $\frac{R_{\max }}{R_{0}}<2$. The UCA destruction only happened once $\frac{R_{\max }}{R_{0}}>2$, although again some of the UCAs exhibited higher amplitude of oscillations. Based on these results, we have chosen the destruction threshold to be equal to two to avoid UCA destruction.

It should be noted that the results of our study at the destruction point also satisfies the criteria developed by Mitchell and Plesset [43]. According to this theoretical study, the bubble is stable if $\frac{R_{\max }}{R_{\min }}<5$ and it is unstable if $\frac{R_{\max }}{R_{\min }}>10$. In our study, the maximum $\frac{R_{\max }}{R_{\min }}$ in the nondestructive regime is $4.11\left(R_{0}=4 \mu \mathrm{m}\right.$, $f_{\mathrm{s}}=0.88 \mathrm{MHz}$ and $P_{\mathrm{A}}=137 \mathrm{kPa}$ ) which is below the limit of this criterion. Thus the identified parameter ranges for nondestructive oscillations in this study also satisfy this criterion of bubble stability.

It should be noted that another potentially better indicator for UCA destruction is the UCA wall velocity. However, there has not been a criterion set based on the UCA wall velocity limit. There have been experimental studies in which the bubble wall velocity has been recorded or estimated during the collapse. We have also compared the maximum simulated UCA wall velocity at the destruction threshold with the published destructive bubble wall velocities. The bubble wall velocities of $-51 \mathrm{~m} / \mathrm{s}$ in [36] ("apparently sufficient to destroy very small bubbles" [36]), $-350 \mathrm{~m} / \mathrm{s}$ in [37] and -144 , -329 and $-456 \mathrm{~m} / \mathrm{s}$ in [44] are reported to be sufficient for bubble destruction. The maximum negative and positive wall velocities in our simulations are -48.92 and $36.47 \mathrm{~m} / \mathrm{s}$ for $\left(R_{0}=1 \mu \mathrm{m}, f_{\mathrm{s}}=5.46 \mathrm{MHz}\right.$ and $\left.P_{\mathrm{A}}=340 \mathrm{kPa}\right)$, which are smaller than the reported velocities for the bubble destruction.

In this paper, it was shown that the main feature of the UCA oscillations driven with $f_{\mathrm{r}}$ is that $R_{n}$ grows monotonically with pressure up to a pressure threshold and beyond which the UCA oscillations undergoes successive period-doubling bifurcations leading to eventual chaotic oscillations. The occurrence of the period 
doubling is concomitant with counterintuitive decrease in the backscatter pressure. This is due to the fact that the magnitude of the UCA contraction decreases with period doubling (Fig. 6), which results in lower wall velocity and acceleration when compared to the case of the period-one oscillation. Thus, in order to maximize the backscatter pressure during nondestructive oscillations, period-one oscillations of full amplitude $\left(R_{n}=2\right)$ are needed.

It was shown that through driving the UCA with $f_{\mathrm{r}}$, radial oscillations of period one with the full amplitude $\left(R_{n}=2\right)$ are not developed. In this regard, application of $f_{\mathrm{s}}$ will be of great advantage. If $f_{\mathrm{s}}$ is optimized correctly, period-one oscillations with full amplitude $\left(R_{n}=2\right)$ can be generated. This results in significant enhancement of the backscattered pressure without the UCA destruction. In addition, because the maximum backscatter pressure occurs at a lower applied acoustic pressure, the background noise is minimized, which may lead to a superior SNR and CTR. Calculation of the maximum possible scattering cross section $\left(\mathrm{SCS}_{\max }\right)$ showed that through optimizing $f_{\mathrm{s}}$, nondestructive $\mathrm{SCS}_{\max }$ can be enhanced up to ninefold.

The backscattered pressure amplitude was calculated using Eq. 3; however, the frequency-dependent attenuation that will occur in tissue was not considered. Thus, high-frequency components that will be preferentially diminished in tissue were persevered. As the main goal of the paper was the study of the dynamical characteristics of the UCA which is independent from attenuation, we have not considered the spreading loss due to attenuation. However, in applications, we may expect a better enhancement in case of the sonication with the pressure-dependent resonance frequency compared to the values predicted here. This is because the pressure-dependent resonance frequency is smaller ( $\sim 30-35 \%$ in this study) compared to the linear resonance frequency; thus the backscatter signal from UCAs experiences less attenuation.

In many investigations, the resonance frequency of the UCAs is experimentally measured through attenuation measurements. The applied pressure amplitude may significantly shift the resonance frequency with larger UCAs experiencing a more drastic effect. The results indicate that in order to increase the efficiency of the UCA applications, either corrections should be made for the measured resonance frequency or the pressure amplitude of the sonication should be chosen higher than the pressure amplitude used for mea- suring the resonance frequency of the UCAs. This is because choosing pressures below the value $\left(P_{\mathrm{s}}\right)$ used in the measurements, the backscattered pressure is significantly reduced (Fig. 4a-d).

The sudden increase in the radial oscillations of the UCA for $P_{\mathrm{A}}>P_{\mathrm{S}}$ may provide advantages to current diagnostic and therapeutic applications of the UCA. One of the diagnostic applications that can benefit from this phenomenon is the amplitude modulation (AM) UCA imaging technique [45-47]. This method is based on the nonlinear increase in the backscatter pressure when pulses of different amplitude are applied. In AM, two pulses are sent to the tissue with the first having twice the amplitude of the second. The backscattered pressure from the second pulse is scaled by multiplying its magnitude by two and it is then subtracted from the backscattered pressure of the first pulse. Due to the linear response of the tissue, the residual from the tissue will be minimal; however there will be a significant residual from the UCAs, which leads to a superior CTR. The sudden increase will be enhanced when increasing the nonlinearity of the UCA system by choosing the half amplitude signal below $P_{\mathrm{s}}$ and the full amplitude above $P_{\mathrm{s}}$.

Another benefit may be in the case of imaging a region of interest (ROI) deep within the body. Because the UCAs are distributed within all the vessels among the pre-target tissue layers, they may shield the signals on the beam path and from the target tissue and UCAs in the ROI. Specifically, this tends to be more problematic in cases of deeper targets. This is because the signal encounters more UCAs on its path to the target and back, and therefore the signal significantly loses its strength. Consequently the ultrasonic beams become attenuated by the superficial pre-focal tissue, which causes the loss of the strength of the signal from the UCAs at the target and thus limits the visualization of the tissue layers at deeper locations $[48,49]$. The accuracy of tissue perfusion measurement is largely affected by this shadowing effect $[48,49]$. To allow accurate quantification, removal of shadowing artifact is crucial $[47,49]$. Near resonance, the attenuation is higher because of increased scattering and energy absorption by the UCAs [18]. In this regard, the imaging procedure can be optimized by using focused transducers that produce the pressures greater than $P_{\mathrm{s}}$ at the ROI (focal region) and less than $P_{\mathrm{s}}$ at the superficial tissue. This will decrease the prefocal shadowing effect (because pre-focal UCAs will be non-resonant) and at the same 
time increases the backscatter at the ROI (the UCAs at the ROI are resonant because the focal pressure is more than $P_{\mathrm{S}}$ ) which can ultimately improve the SNR and CTR.

One of the therapeutic applications that can benefit from this nonlinear behavior of UCAs is the microbubble-enhanced drug delivery. In drug delivery, UCA oscillations are used to enhance the permeability of the cell to the drug [15]. However, multiple scattering of the UCAs coming from pre-focal regions will attenuate the ultrasonic beams and have undesirable effects on the healthy (non-targeted) tissue while also distorting the focus at the target tissue [50]. Because of the steep pressure gradient of the therapy transducers, pressures above $P_{\mathrm{s}}$ can be generated at the target, while the pressure in the surrounding tissue can be kept below $P_{\mathrm{s}}$. This way, the microbubbles in the non-focal surrounding tissue will oscillate below resonance and therefore the pre-focal scattering effects and attenuations are minimized. In addition, the microbubble activity will be enhanced in the focal region, due to enhanced oscillations in pressure-dependent resonance regime. This can lead to a more effective and precise treatment and enhanced safety.

This work considered single-size microbubbles. However, location of the saddle-node bifurcation is highly size dependent. Thus, in case of a polydisperse solution of microbubbles (which is generally the case in clinical investigations), only a fraction of microbubble sizes will be active in the pressure-dependent resonance regime. One possible way to partially take advantage of the pressure-dependent resonance can be to choose a frequency which excites the majority of the microbubbles around the resonance peak. The other method could be the use of centrifugation techniques to stack the micobubbles in narrow size ranges [51], then the driving frequency can be chosen so that it drives the majority of each stack into pressure- dependent resonance regime. Through using monodisperse microbubbles $[23,52,53]$, one may fully take advantage of exciting all the microbubbles in the pressuredependent resonance regime.

Another factor that should be considered is the microbubble growth due to rectified diffusion. In applications where long high-amplitude pulses are used for sonication, the effect of rectified diffusion may become significant. This can displace the pressure for saddlenode bifurcation in the microbubble system as the microbubble can grow beyond the active size range due to rectified diffusion. One possible way to solve this problem is modifying the pulsing sequences by choosing a suitable combination of "pulse on time"/"pulse off time" strategy [54]. The duration of the pulse on time may ensure the microbubbles are still in effective pressure- dependent resonance regime, while the "pulse off" time duration may ensure that there is enough partial dissolution of microbubbles. The "pulse off" time duration may allow reactivation of the microbubbles which have grown beyond the active size range at the beginning of the subsequent pulse [54].

\section{References}

1. Parlitz, U., Englisch, V., Scheczyk, C., Lauterborn, W.: Bifurcation structure of bubble oscillators. J. Acoust. Soc. Am. 88(2), 106177 (1990)

2. Lauterborn, W., Suchla, E.: Bifurction straucture in a model of acoustic turbulence. Phys. Rev. Lett. 53(24), 2304-2307 (1984)

3. Esche, R.: Untersuchungder schwingungs kavitation in ussigkeiten. Acustica 2, 20818 (1952)

4. Luterborn, W., Cramer, L.: Subharmonic route to chaos observed in acoustics. Phys. Rev. Lett. 47(20), 14458 (1981)

5. Lauterborn, W., Koch, A.: Holographic observation of period-doubled and chaotic bubble oscillations in acoustic cavitation. Phys. Rev. A 35(4), 19746 (1987)

6. Holt, R.G., Gaitan, D.F., Atchley, A.A., Holzfuss, J.: Chaotic sonoluminescence. Phys. Rev. Lett. 72(9), 13769 (1994)

7. Hegedűs, F., Hős, C., Kullmann, L.: Stable period 1, 2 and 3 structures of the harmonically excited Rayleigh-Plesset equation applying low ambient pressure. IMA J. Appl. Math. 78(6), 1179-1195 (2013)

8. Macdonald, C.A., Gomatam, J.: Chaotic dynamics of microbubbles in ultrasonic elds. Proc. IMechE. 220, 333 343 (2006)

9. Behnia, S., Jafari, A., Soltanpoor, W., Jahanbakhsh, O.: Nonlinear transitions of a spherical cavitation bubble. Chaos, Solitons Fractal 41, 818-828 (2009)

10. Behnia, S., Jafari, A., Soltanpoor, W., Sarkhosh, L.: Towards classificationof the bifurcation structure of a spherical cavitation bubble. Ultrasonics 49, 605-610 (2009)

11. Sojahrood, A.J., Kolios, M.C.: Classification of the nonlinear dynamics and bifurcation structure of ultrasound contrast agents excited at higher multiples of their resonance frequency. Phys. Lett. A 376(33), 2222-2229 (2012)

12. Cosgrove, D.: Ultrasound contrast agents: an overview. Eur. J. Radiol 60, 324-330 (2006)

13. Suslick, K.S.: Sonochemistry. In: Kroschwitz, J. (ed.) KirkOthmer concise encyclopedia of chemical technology, 4th edn., pp. 1867-1868. Wiley, New York (1999)

14. Suslick, K.S., Price, J.G.: Application of ultrasound to materials chemistry. Ann. Rev. Mater. Sci. 29, 295-326 (1999)

15. Ferrara, K., Pollard, R., Borden, M.: Ultrasound microbubble contrast agents: fundamentals and application to gene and drug delivery. Ann. Rev. Biomed. Eng. 9, 415-447 (2007) 
16. Nhan, T., et al.: Drug delivery to the brain by focused ultrasound induced bloodbrain barrier disruption: quantitative evaluation of enhanced permeability of cerebral vasculature using two-photon microscopy. J. Control. Release 172, 274280 (2013)

17. Stieger, S.S., et al.: Imaging of angiogenesis using Cadence contrast pulse sequencing and targeted contrast agents. Contrast Media Mol. Imaging 3(1), 9-18 (2008)

18. Deng, C.X., Lizzi, F.L.: A review of the physical phenomena associated with ultrasonic contrast agents and illustrative clinical applications. Ultrasound Med. Biol. 28(3), 277-286 (2002)

19. Schrope, B.A., Newhouse, V.L., Uhlendorf, V.: Simulated capillary blood flow measurement using a nonlinear ultrasonic contrast agent. Ultrason. Imaging 14, 134-158 (1992)

20. Lauterborn, W.: Numerical investigation of nonlinear oscillations of gas bubbles in liquids. J. Acoust. Soc. Am. 59(2), 283-293 (1976)

21. MacDonald, C.A., Sboros, V., Gomatam, J., Pyec, S.D., Moran, C.M., McDicken, W.N.: A numerical investigation of the resonance of gas filled microbubbles: resonance dependence on acoustic pressure amplitude. Ultrasonics $\mathbf{4 3}$, 113-122 (2004)

22. Doinikov, A.A., Haac, J.F., Dayton, P.A.: Resonance frequencies of lipid-shelled microbubbles in the regime of nonlinear oscillations. Ultrasonics 49(2), 263-268 (2009)

23. Gong, Y., Cabodi, M., Porter, T.: Pressure-dependent resonance frequency for lipid-coated microbubbles at low acoustic pressures. In: IEEE International ultrasonics symposium proceedings (2010). doi:10.1109/ULTSYM.2010. 0488

24. Helfield, B.L., Goertz, D.E.: Nonlinear resonance behavior and linear shell estimates for definity and micromarker assessed with acoustic microbubble spectroscopy. J. Acoust. Soc. Am. 133, 1158-1168 (2013)

25. Hoff, L., Sontum, P.C., Hovem, J.M.: Oscillations of polymeric microbubbles: effect of the encapsulating shell. J. Acoust. Soc. Am. 107(4), 2272-2280 (2000)

26. Hilgenfeldt, S., Lohse, D., Zomack, M.: Sound scattering and localized heat deposition of pulse-driven microbubbles. J. Acoust. Soc. Am. 107(6), 3530-3539 (2000)

27. Kvikliene, A., et al.: Modeling of nonlinear effects and the response of ultrasound contrast micro bubbles: simulation and experiment. Ultrasonics 42, 301-307 (2004)

28. Church, C.C.: The effects of an elastic solid surface layer on the radial pulsations of gas bubbles. J. Acous. Soc. Am. 97(3), 1510-1521 (1995)

29. Morgan, K.E., Allen, J.S., Dayton, P.A., Chomas, J.E., Klibanov, A.L., Ferrara, K.W.: Experimental and theoretical evaluation of microbubble behavior: effect of transmitted phase and bubble size. IEEE Trans. Ultrason. Ferroelectr. Freq. Control 47(6), 1494-1509 (2000)

30. Hegedús, F.: Stable bubble oscillations beyond Blake's critical threshold. Ultrasonics 54(4), 1113-1121 (2014)

31. Flynn, H.G., Church, C.C.: Transient pulsations of small gas bubbles in water. J. Acoust. Soc. Am. 84, 985-998 (1988)

32. Akhatov, I., Gumerov, N., Ohl, C.D., Parlitz, U., Lauterborn, W.: The role of surface tension in stable single-bubble sonoluminescence. Phys. Rev. Lett 78, 227-230 (1997)
33. Flynn, H.G.: Cavitation dynamics II. Free pulsations and models of cavitation bubbles. J. Acoust. Soc. Am. 58, 11601170 (1975)

34. Leighton, T.G.: The Acoustic bubble. Academic Press Limited, London, UK (1994)

35. Apfel, R.E.: Some new results on cavitation threshold prediction and bubble dynamics. In: Lauterborn, W. (ed.) Cavitation and inhomogeneities in underwater acoustics, pp. 7983. Springer, Berlin (1980)

36. Chomas, J.E., Dayton, P.A., May, D., Ferrara, K.: Threshold of fragmentation for ultrasonic contrast agents. J. Biomed. Opt. 6(2), 141-150 (2001)

37. Chomas, J.E., Dayton, P.A., May, D., Klibanov, A., Ferrara, K.: Optical observation of contrast agent destruction. Appl. Phys. Lett. 77, 1056 (2000)

38. Bloch, H., Wan, M., Dayton, P.A., Ferrara, K.: Optical observation of lipid- and polymer-shelled ultrasound microbubbles. Appl.Phys. lett. 84, 631 (2004)

39. Chomas, J., Dayton, P., May, D., Ferrara, K.: Nondestructive subharmonic imaging. IEEE Trans. Ultrason. Ferroelectr. Freq. Control 9(7), 883-892 (2002)

40. Santin, M.D., King, D.A., Foiret, J., Haak, A., O’Brien Jr, W.D., Bridal, S.L.: Encapsulated contrast microbubble radial oscillation associated with postexcitation pressure peaks. J. Acoust. Soc. Am. 127, 1156 (2010)

41. King, D.A., W O'Brien Jr, W.D.: Comparison between maximum radial expansion of ultrasound contrast agents and experimental postexcitation signal results. J. Acoust. Soc. Am. 129, 114 (2011)

42. King, D.A., Malloy, M.J., Roberts, A.C., Haak, A., Yoder, C.C., O'Brien, W.D.: Determination of postexcitation thresholds for single ultrasound contrast agent microbubbles using double passive cavitation detection. J. Acoust. Soc. Am. 127, 3449 (2010)

43. Plesset, M.S., Mitchell, T.P.: On the stability of the spherical shape of a vapor cavity in a liquid. Quart. Appl. Math. 13419-430 (1956)

44. Church, C.C., Cartensen, E.: "Stable" inertial cavitation. Ultrasound Med. Biol. 27(10), 1435-1437 (2001)

45. Philips, P., Gardner, E.: Contrast-agent detection and quantification. Eur. Radiol. Suppl. 14(8), 4-10 (2004)

46. Brock-Fisher, A.G., Poland, M., Rafter, P.: Means for increasing sensitivity in nonlinear imaging systems. US Patent 5577505 (1996)

47. Eckersley, R.J., Chin, C.T., Burns, P.N.: Optimizing phase and amplitude modulation schemes for imaging microbubble contrast agents at low acoustic power. Ultrasound Med. Biol. 6(31), 213-219 (2005)

48. Bos, L.J., Piek, J.J., Spaan, J.A.: Effects of shadowing on the time-intensity curves in contrast echocardiography: a phantom study. Ultrasound Med. Biol. 22(2), 217-227 (1996)

49. Tang, M.X., Eckersley, R.J., Noble, J.A.: Regularized estimation of contrast agent attenuation to improve the imaging of microbubbles in small animal studies. Ultrasound Med. Biol. 34(6), 938-948 (2008)

50. Coussios, C.C., Farny, C.H., Ter Haar, G., Roy, R.A.: Role of acoustic cavitation in the delivery and monitoring of cancer treatment by high-intensity focused ultrasound (HIFU). Int. J. Hyperth. 23(2), 105-120 (2007) 
51. Feshitan, J.A., Chen, C.C., Kwan, J.J., Borden, M.A.: Microbubble size isolation by differential centrifugation. J. Colloid Interface Sci. 329(2), 316-324 (2009)

52. Gong, Yanjun, Cabodi, Mario, Porter, Tyrone M.: Acoustic investigation of pressure-dependent resonance and shell elasticity of lipid-coated monodisperse microbubbles. Appl. Phys. Lett. 104(7), 074103 (2014)

53. Parrales, M.A., et al.: Acoustic characterization of monodisperse lipid-coated microbubbles: relationship between size and shell viscoelastic properties. J. Acoust. Soc. Am. 136(3), 1077 (2014)

54. Hauptmann, M., Struyf, H., Gendt, De, Glorieux, C., Brems, S.: Importance of bubble size control in ultrasonic surface cleaning by pulsed high-frequency sound fields. ECS J. Solid State Sci. Technol. 3(1), N3032-N3040 (2014) 